\title{
Optical anisotropy in vertically coupled quantum dots
}

Yu, Ping; Langbein, Wolfgang Werner; Leosson, Kristjan; Hvam, Jørn Märcher; Ledentsov, N. N.; Bimberg, Dieter; Ustinov, V. M.; Egorov, A. Yu.; Zhukov, A. E.; Tsatsul'nikov, A. F. Total number of authors: 11

Published in:

Physical Review B Condensed Matter

Link to article, DOI:

10.1103/PhysRevB.60.16680

Publication date:

1999

Document Version

Publisher's PDF, also known as Version of record

Link back to DTU Orbit

Citation (APA):

Yu, P., Langbein, W. W., Leosson, K., Hvam, J. M., Ledentsov, N. N., Bimberg, D., Ustinov, V. M., Egorov, A. Y., Zhukov, A. E., Tsatsul'nikov, A. F., \& Musikhin, Y. G. (1999). Optical anisotropy in vertically coupled quantum dots. Physical Review B Condensed Matter, 60(24), 16680-16685. https://doi.org/10.1103/PhysRevB.60.16680

\section{General rights}

Copyright and moral rights for the publications made accessible in the public portal are retained by the authors and/or other copyright owners and it is a condition of accessing publications that users recognise and abide by the legal requirements associated with these rights.

- Users may download and print one copy of any publication from the public portal for the purpose of private study or research.

- You may not further distribute the material or use it for any profit-making activity or commercial gain

- You may freely distribute the URL identifying the publication in the public portal 


\title{
Optical anisotropy in vertically coupled quantum dots
}

\author{
P. Yu, ${ }^{*}$ W. Langbein ${ }^{\dagger}$ K. Leosson, and J. M. Hvam \\ Research Center COM, The Technical University of Denmark, DK-2800 Lyngby, Denmark \\ N. N. Ledentsov and D. Bimberg \\ Institut für Festkörperphysik, Technische Universität Berlin, D-10623 Berlin, Germany
}

\begin{abstract}
V. M. Ustinov, A. Yu. Egorov, A. E. Zhukov, A. F. Tsatsul'nikov, and Yu. G. Musikhin
A. F. Ioffe Physical-Technical Institute, 194021 Saint Petersburg, Russia
\end{abstract}

(Received 12 October 1998; revised manuscript received 10 June 1999)

\begin{abstract}
We have studied the polarization of surface and edge-emitted photoluminescence (PL) from structures with vertically coupled $\mathrm{In}_{0.5} \mathrm{Ga}_{0.5} \mathrm{As} / \mathrm{GaAs}$ quantum dots (QD's) grown by molecular beam epitaxy. The PL polarization is found to be strongly dependent on the number of stacked layers. While single-layer and 3-layer structures show only a weak TE polarization, it is enhanced for 10-layer stacks. The 20-layer stacks additionally show a low-energy side-band of high TE polarization, which is attributed to laterally coupled QD's forming after the growth of many layers by lateral coalescence of QD's in the upper layers. While in the single, 3- and 10-layer stacks, both TE polarized PL components are stronger than the TM component, the [110] TE component is weaker than the TM component in the 20-layer stack. This polarization reversal is attributed to an increasing vertical coupling with increasing layer number due to increasing dot size.
\end{abstract}

[S0163-1829(99)05747-1]

\section{INTRODUCTION}

In the strained $\operatorname{In}_{x} \mathrm{Ga}_{1-x}$ As semiconductor system, pyramid or disc-shaped quantum dots (QD's) of $\operatorname{In}_{x} \mathrm{Ga}_{1-x}$ As are formed on top of the initial planar wetting layer during growth on GaAs in the Stranski-Krastanow growth mode. ${ }^{1-3}$ Although the individual dots have zero-dimensional properties, dot ensembles show an inhomogeneous broadening of the emission lines due to size fluctuations. This size inhomogeneity reduces the advantages of the zero-dimensionality. Recently, multiple stacked layers of QD's with GaAs or $\mathrm{Al}_{x} \mathrm{Ga}_{1-x} \mathrm{As}$ spacers were reported, which show self alignment of the QD's into vertical columns. ${ }^{4-7}$ During the stacking process, the shape uniformity of the dots in the higher layers is improved and thereby the inhomogeneity of the optical transitions is decreased. These vertically coupled quantum dots (VCQD's) have been characterized by scanning tunneling microscopy, high-resolution x-ray diffraction and transmission electron microscopy (TEM). ${ }^{7,8}$ The vertical alignment is caused by the strain fields of the lower QD layer extending into the barrier material. Due to the strong vertical coupling between the dots, the electronic states can acquire a wire-like character.

In semiconductor quantum wires, the lowest optical transition is in general polarized along the wire direction, as has been observed experimentally and analyzed theoretically. ${ }^{9-14}$ Accordingly, the photoluminescence (PL) polarization in VCQD's should be modified by the vertical correlation. The polarization properties of the VCQD's are also of practical interest since they influence the optical gain in laser diodes using VCQD's as an active material. However, there is to our knowledge no report about the polarization anisotropy of VCQD's in the literature. We will present here the optical polarization anisotropy of single-layer QD's and stacked
VCQD's to analyze the changes of the optical polarization of the PL emission induced by the vertical stacking.

\section{EXPERIMENTAL DETAILS}

The samples were grown on (001) Si-doped GaAs substrates by elemental source molecular beam epitaxy (MBE), and contain $1,3,10$, and 20 layers of $\operatorname{In}_{0.5} \mathrm{Ga}_{0.5}$ As QD's deposited at $485^{\circ} \mathrm{C}$ and separated by $5 \mathrm{~nm}$-thick GaAs barriers. The QD's have pyramidal shape with a base length of about $18 \mathrm{~nm}$, a height of about $5 \mathrm{~nm}$, and an average lateral separation of $55 \mathrm{~nm}$. The base sides of the pyramidal dots are oriented along the [100] and [010] directions. The vertical alignment of the QD's was identified in TEM investigations. ${ }^{7}$ Figure 1 shows schematically the structure of the VCQD's. Details about growth conditions and sample structure are re-

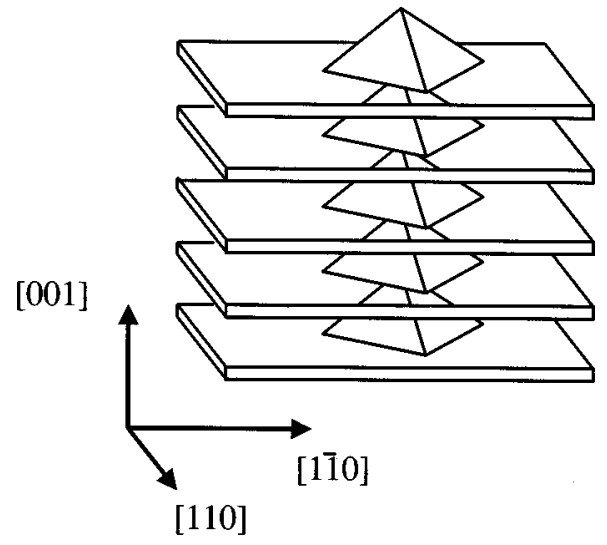

FIG. 1. Schematic arrangement of vertically coupled quantum dots and wetting layers together with the axes referred to in the text. Only 5 layers are shown for simplicity. 
ported in Ref. 15. The samples were mounted in a closedcycle cryostat at a temperature varied between 10 and $300 \mathrm{~K}$. The PL was excited by a He-Ne laser, and detected spectrally resolved by a monochromator and a cooled Ge detector. The PL was transmitted through a $\lambda / 2$ plate and a polarizer before entering into the detection. The direction of the polarizer was chosen to fit the maximum efficiency of the monochromator. The $\lambda / 2$ plate was rotated at about $10 \mathrm{~Hz}$, and four times the rotation frequency was used as a reference of a lock-in amplifier analyzing the detector signal. The broadband $\lambda / 2$ plate that we used in the experiments has a wavelength range covering most of PL bands in the samples. However, some PL bands are located at the edge of wavelength limit of the $\lambda / 2$ plate, where the polarization anisotropy values have been recalibrated. We define the PL polarization anisotropy as $P$ $=\left(I_{\|}-I_{\perp}\right) /\left(I_{\|}+I_{\perp}\right)$, where $I_{\|}$is the vertically polarized intensity and $I_{\perp}$ is the horizontally polarized intensity in the laboratory coordinate system. The polarization anisotropy of the PL was found to be oriented in the three perpendicular directions [110], [1-10], and [001] relative to the sample as shown in Fig. 1. The sample orientation was determined experimentally from the GaAs cleavage planes and the orientation of oval surface defects. For the light propagating in the layer planes, we define $I_{\|}\left(I_{\perp}\right)$ as the PL intensity polarized in (normal to) the surface plane. For the light propagating in [001] direction (surface emission), $I_{\|}$and $I_{\perp}$ are polarized along the [110] and [1-10] directions, respectively. The polarization anisotropy and the PL were measured simultaneously by detecting direct current (DC) and alternating current (AC) signals, and was calibrated using an additional polarizer in front of the $\lambda / 2$ plate. This arrangement effectively eliminates influences of the grating efficiency and PL intensity fluctuations on the polarization anisotropy. TEM studies were performed by using a JEOL JEM 4000 Ex microscope operated at an acceleration voltage of $400 \mathrm{kV}$. The TEM images were taken under conditions far away from the exact Bragg reflection to minimize masking of the true island shape by strain fields.

\section{EXPERIMENTAL RESULTS}

Figure 2 shows typical plan-view TEM images of VCQD samples. The contrast is mainly due to strain fields. The TEM images reveal that the dots are slightly elongated along [1-10] direction in the sample with a small number of stacks. In the sample with a large number of stacks, the TEM image shows a lateral ordering of QD's at the topmost layer. These laterally coupled QD's are formed after the growth of many layers by lateral coalescence of QD's in the upper layers.

The solid lines in Fig. 3 show the PL spectra at $10 \mathrm{~K}$ of 1-, 10-, and 20-layer QD samples. The most intense PL band of each sample is assigned to the ground state transitions of the VCQD's. The line-widths between 44 and $56 \mathrm{meV}$ are typical inhomogeneous broadenings of dot ensembles. The redshift of the peak position and the reduction of the line width from the 1-layer sample to the 20-layer sample result from the vertical coupling. ${ }^{5}$ The PL of the 3-layer sample (not shown in the figure) is similar to the PL of the singlelayer sample except for slight differences in the peak position and line-width. The PL of GaAs is visible at $1.49 \mathrm{eV}$ (carbon impurity PL). The PL spectrum in the single-layer

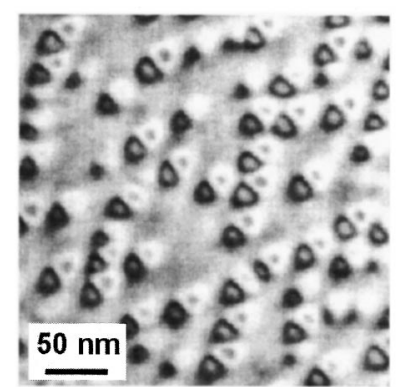

(a)

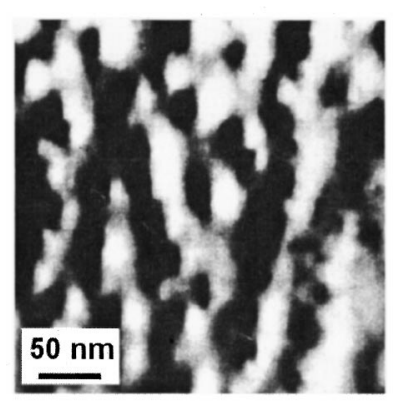

[100]

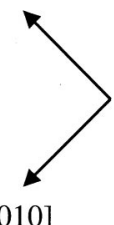

(b)

FIG. 2. Plan-view TEM taken under conditions far away from exact Bragg reflection. (a) 3-layer sample, (b) 20-layer sample.

sample shows a symmetric band centered at $1.32 \mathrm{eV}$ when the excitation density is lower than $1.0 \mathrm{~W} / \mathrm{cm}^{2}$. At higher excitation densities of $1-10 \mathrm{~W} / \mathrm{cm}^{2}$, higher transitions can be observed after the saturation of the ground states by the initial capture and relaxation of carriers into the QD's. The VCQD's show a slightly asymmetric PL band as a result of the coupling within a dot stack, quenching the PL at higher energy by exciton relaxation within the stacks. In the 10layer and 20-layer samples, no excited-state transition is observed at $10 \mathrm{~K}$ below the excitation density of $10 \mathrm{~W} / \mathrm{cm}^{2}$, indicating a higher saturation intensity.

The PL spectrum of the 20-layer sample shows in addition to the main PL band a broad side band located at the low-energy side of the VCQD ground-state transition. According to Ref. 16, the emission band with a peak position of $1.13 \mathrm{eV}$ is due to ground state PL of laterally coupled quantum dots (LCQD's), forming in VCQD's with a large number of stacks by the coalescence of stacks that was proven by the TEM images. The LCQD PL intensity was observed to increase with temperature, as in our results. The PL intensity of the LCQD's stays lower than that of the VCQD's up to room temperature in our sample [see solid curves in Fig. 4(b)], which is different than reported in Ref. 16. The lower saturation intensity of the LCQD's of $1 \mathrm{~W} / \mathrm{cm}^{2}$ compared to that of the VCQD's shows the relatively low density of the LCQD's.

Also in the 10-layer sample, a doublet structure in the PL spectra at room temperature can be seen [Fig. 4(a)], which changes relative intensity with temperature. This structure differs from the one observed in the 20-layer sample by the energy separation and the polarization anisotropy. Its separation of $50 \mathrm{meV}$ is smaller than the separation of $130 \mathrm{meV}$ between VCQD and LCQD in the 20-layer sample.

The dashed lines in Fig. 4 show the polarization anisotropy observed in the [001] direction. The polarization of the 

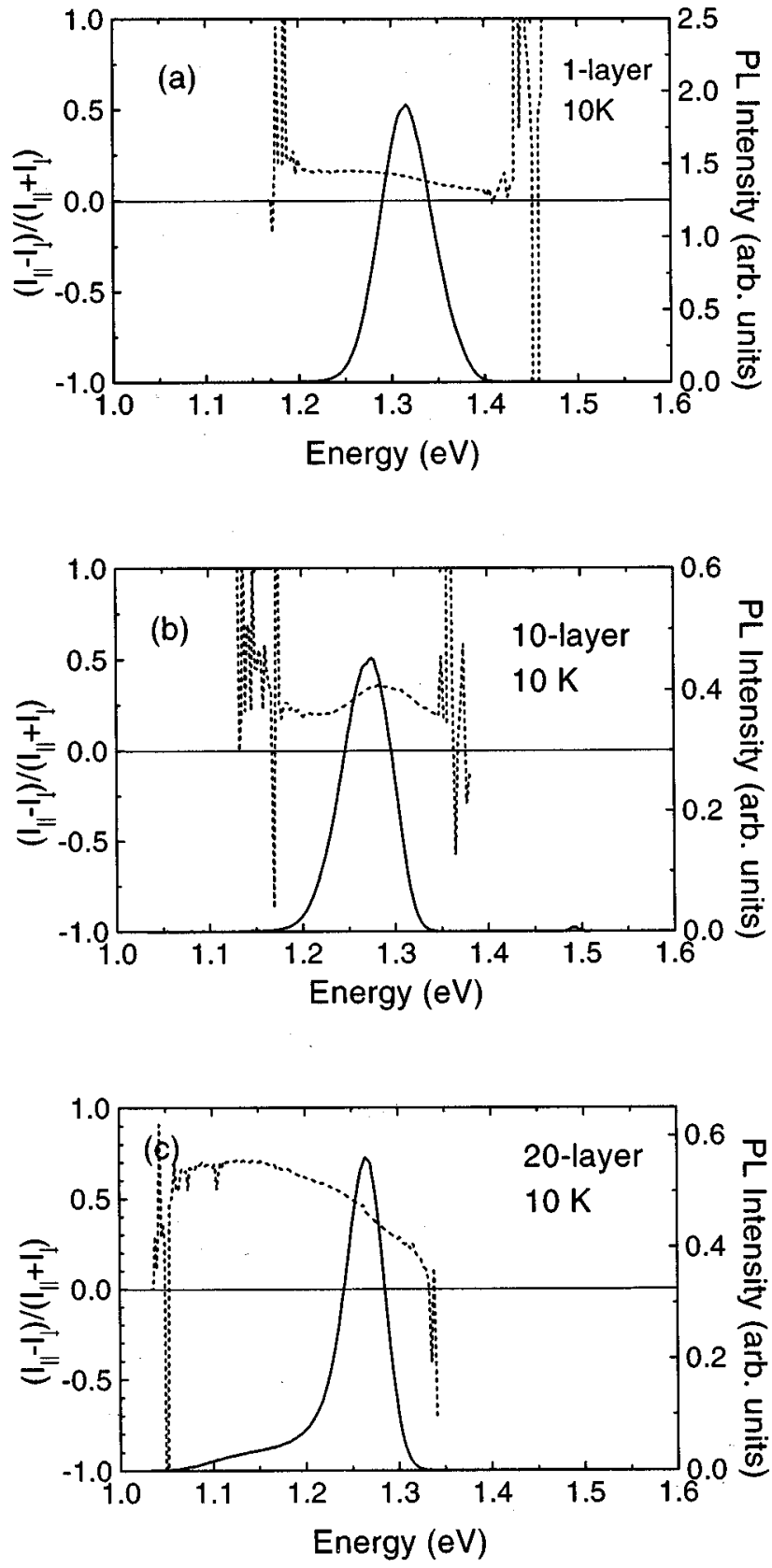

FIG. 3. Photoluminescence data at an excitation intensity of 1 $\mathrm{W} / \mathrm{cm}^{2}$ and $10 \mathrm{~K}$ detected along the [001] direction. Solid lines: Intensity spectra. Dashed lines: polarization anisotropy spectra. (a) Single-layer sample, (b) 10-layer sample, and (c) 20-layer sample.

PL is not influenced by the excitation polarization since the excitation photon energy is above the band gap of GaAs, and the initial polarization is lost during the carrier relaxation into the QD's. No pronounced difference can be seen for different pumping intensities. The polarization anisotropy spectra are limited to wavelengths of large PL intensity. At $1.49 \mathrm{eV}, P=0$ is observed due to the isotropic emission of the bulk GaAs substrate. In the single-layer and 3-layer QD's, the polarization spectra show a small anisotropy $(P$ $\sim 0.16$ ), and a rise of $P$ with decreasing photon energy, indicating that the larger dots in the ensemble have a larger anisotropy. For the multiply stacked QD's, the in-plane polarization anisotropy of the ground state transitions of the VCQD's is around $P \sim 0.4$. The LCQD's of the 20-layer
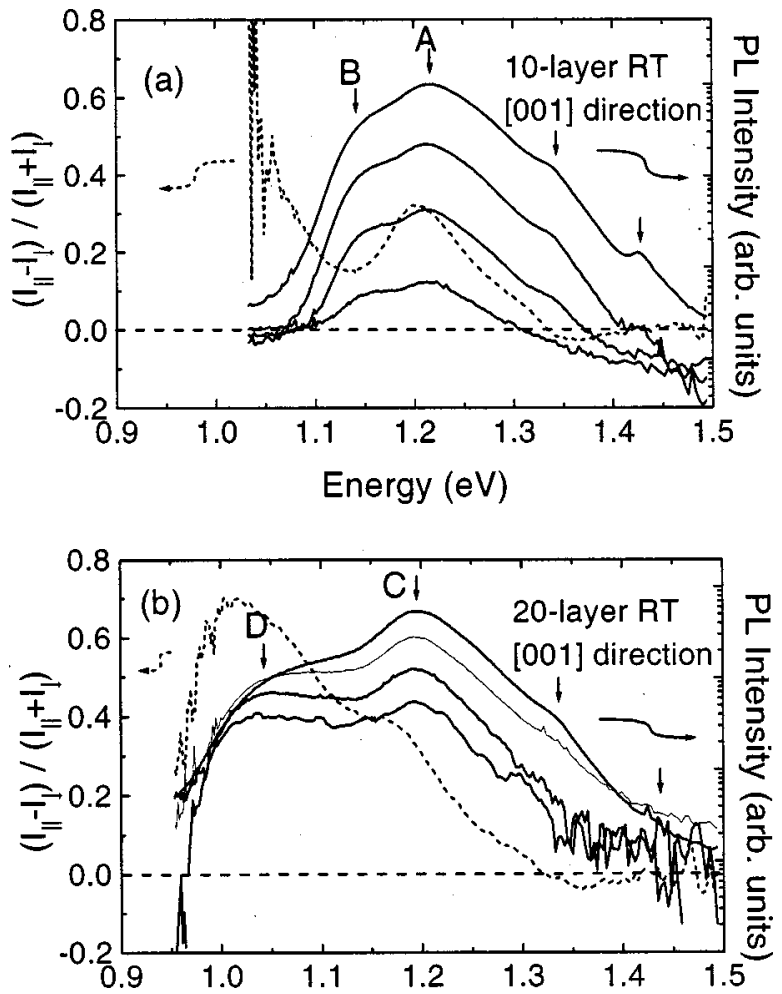

FIG. 4. Photoluminescence data at room temperature detected along the [001] direction for the 10-layer sample (a) and the 20layer sample (b). Solid lines: Intensity spectra measured at 0.03 , $0.1,0.3$, and $1 \mathrm{~W} / \mathrm{cm}^{2}$ from bottom to top. Dashed lines: Polarization anisotropy spectra measured at $1 \mathrm{~W} / \mathrm{cm}^{2}$.

sample show a high anisotropy of $P \sim 0.7$. In the 10-layer sample, however, the lower-energy peak shows a lower polarization anisotropy than the VCQD's peak. Additionally, although the PL emission at the photon energy position of the LCQD's can hardly be observed, the anisotropy spectrum rises again at this energy. This is evidence for the existence of a small amount of the LCQD's also in the 10-layer sample, which are not dominating the PL as in the 20-layer structure. At room temperature, the polarization anisotropy acquires additional features at the high-energy wing of the main PL-band. Simultaneously, higher states can be observed in the PL intensity. These higher states have in the ensemble average a smaller polarization anisotropy, which even changes sign in the energy region around the wetting layer transition (around $1.33 \mathrm{eV}$ at room temperature).

To elaborate further on the origin of the doublet structure in the stacked samples, we show in Fig. 5 the polarization anisotropy spectra of the 10-layer and the 20-layer samples, detected in [110] and [1-10] directions, respectively. The spectra were obtained exciting and detecting at the sample edges. The edge emission was spatially selected in near and far field to suppress PL emission from the [001] sample surface, which has a different propagation direction inside the sample. To visualize the data better, we display in Fig. 6 the intensity ellipses in the three main directions for different PL bands indicated in Figs. 4 and 5. The relative intensities at the fixed energy positions marked in Figs. 4 and 5 are given by $I_{\|} / I_{\perp}=(1+P) /(1-P)$. In the [001] direction, the intensity ellipses show a dominating [1-10] polarization for all samples and bands. In the [110] direction, the 10-layer 

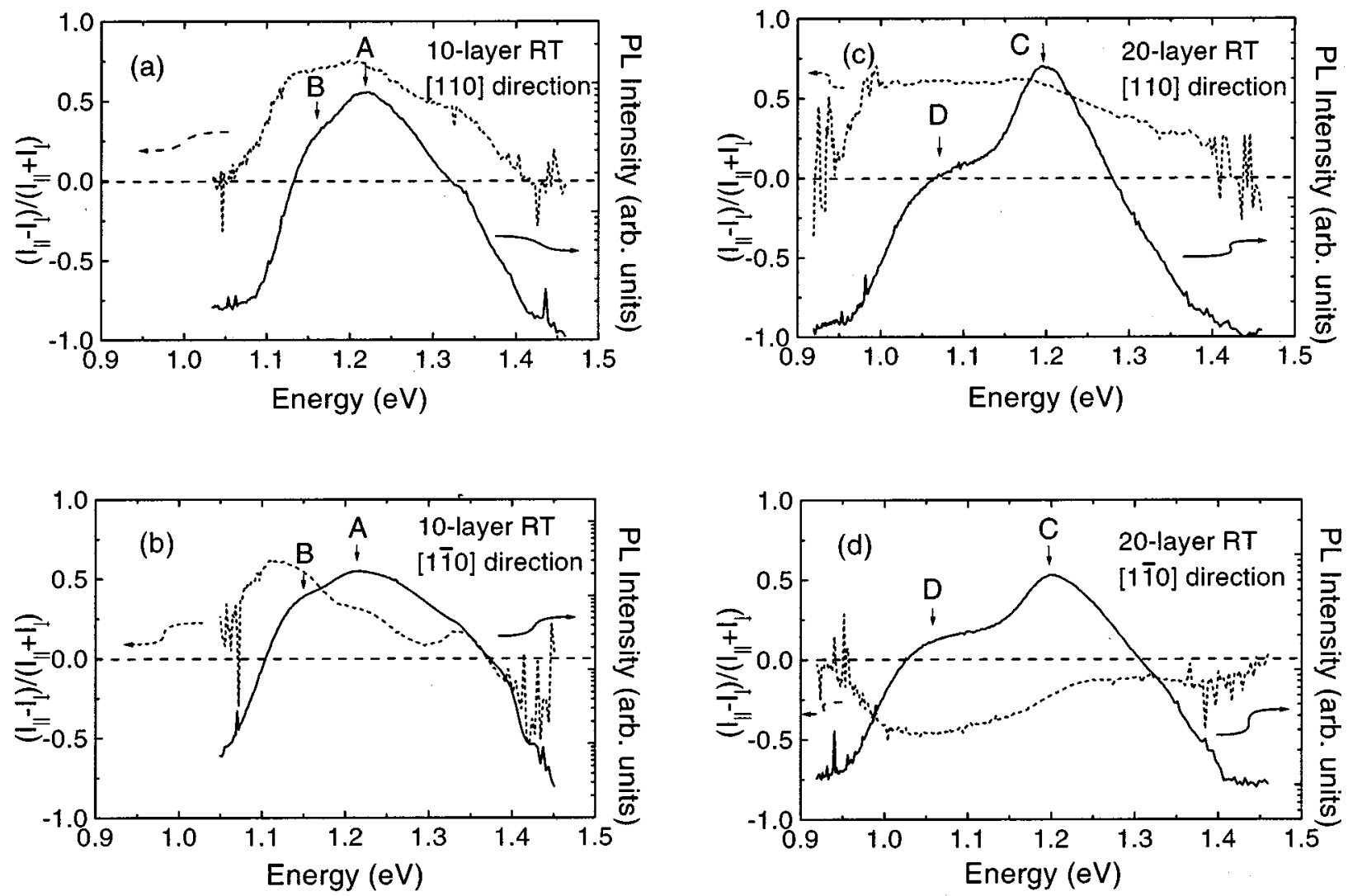

FIG. 5. Photoluminescence intensity and polarization anisotropy spectra detected along the [110] and [1-10] directions. (a) and (b) 10-layer sample, (c) and (d) 20-layer sample. Experimental conditions as in Fig. 3.

sample shows a large $P$ up to 0.75 , i.e., the emission is almost linearly polarized in the sample surface, which is a TE polarization in a waveguide. The 20-layer sample is qualitatively comparable, but with quantitatively smaller polariza-

10-layer sample 20-layer sample
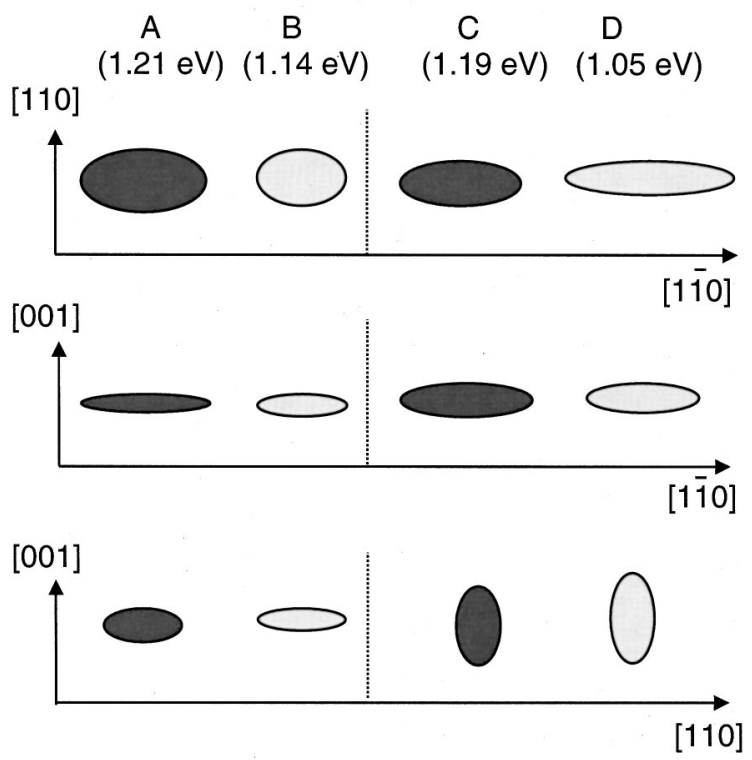

FIG. 6. Photoluminescence intensity ellipses (axis length proportional to intensity) for fixed photon energy positions marked in Figs. 3 and 4 in the projections along the [001], [110], and [1-10] directions. tions. For the [1-10] direction (lowest row in Fig. 6), the two VCQD samples show a qualitatively different behavior. While the 10-layer sample is still TE polarized, the polarization changes to TM polarization in the 20-layer sample. This is important for the application of this material in a laser structure, and shows, that in the 20-layer sample the electronic states are strongly elongated along the growth direction due to the vertical coupling.

\section{DISCUSSION}

Comparable measurements on single-layer pure InAs/ GaAs pyramid QD's with base length along [100] and [010] directions show an in-plane polarization anisotropy of less than $1 \%$, in agreement with the results reported in Ref. 18. On the other hand, a polarization anisotropy of optical transitions has been observed in a single-layer $\operatorname{In}_{x} \mathrm{Ga}_{1-x} \mathrm{AsQD}$ 's grown on GaAs [001] substrate, which have their base sides along [1-10] or [110] directions due to a slight difference in the growth conditions. ${ }^{17-19}$ The optical anisotropy was attributed to shape anisotropy since the bases were found to be slightly elongated along the [1-10] direction in plan view TEM. Single-layer InAs quantum dots grown on GaAs(311)A substrates ${ }^{20}$ showed a PL polarization along the [-233] direction, according to their arrow shape related to the corrugation of the $\operatorname{GaAs}(311) A$ substrate.

Although an exact experimental determination of the shape of the self-assembled QD's is still controversial, different shapes of quantum dots such as pyramids with different facets, lens shaped and round shaped have been verified 
by using high-resolution TEM (HREM). In the $\operatorname{In}_{0.5} \mathrm{Ga}_{0.5} \mathrm{As}$ samples under investigation, HREM indicates that the QD's have a pyramidal shape, ${ }^{7,21}$ and plan-view TEM reveals bases with facets in the [100] and [010] directions, and elongated slightly in [1-10] direction. During the growth of the VCQD samples, the QD's in the initial layer elongate slightly along [1-10] direction. Accordingly, the optical polarization should be along [1-10] direction. After growth of the first QD sheet, indium segregates during overgrowth and contributes to the formation of the second QD sheet, leading to a size increase of QD's in the upper layers. The anisotropic surface diffusion of indium leads to an elongation of the QD's. For a large number of layers, some VCQD stacks merge laterally along the [1-10] direction to form LCQD's. The high-polarization anisotropy of the ground-state transition in the LCQD's comes from their shape asymmetry.

In order to explain the observed polarization properties theoretically, one needs to consider the microscopic details of the strain, valence-band mixing, and the change in the effective masses due to the strain. A number of papers have reported calculations of the energy levels and wave functions of pyramidal quantum dots, typically using a multiband $k \cdot p$ method. In the calculations, the bulk valence band is split into light hole (lh), heavy hole (hh), and spin-orbit (so) band via size quantization (quantum confinement) and uniaxial strain. These three bands have different polarization properties, with the consequence that the lowest confined state is, in general, polarized along the direction of weakest confinement. This explains the polarization anisotropy in arrow-shaped InAs QD's grown on GaAs(311)A substrate, ${ }^{20}$ while square base QD's have isotropic in-plane oscillator strengths. In-plane anisotropy can also be attributed to piezoelectric effects. A theoretical consideration of these strongly strained systems should include the piezoelectric effects, which are present in the zinc-blende structures due to the missing inversion symmetry, and disturb the cubic symmetry of the $k \cdot p$ approximation. In strained-layer superlattices these fields are along the growth axis, leading to a tilting of the band structure. ${ }^{22-25}$ In the QD's, the missing translation symmetry allows for piezoelectric fields along all three directions, with their values given by the local shear strain distribution. Calculations of the electronic structure of InAs/ GaAs quantum dots including piezoelectric effects ${ }^{3}$ show that the shear strain and the piezoelectric charge are close to the QD edges, and the piezoelectric fields are mainly located outside of the QD's. The influence of these piezoelectric fields increases with size, while the quantization decreases. Especially for the $\operatorname{In}_{x} \mathrm{Ga}_{1-x} \mathrm{As} / \mathrm{GaAs}$ case, the reduction of the strain with increasing gallium content is nearly outweighed by the increasing piezoelectricity of GaAs compared to InAs. This points to an important influence of the piezoelectric fields in $\mathrm{In}_{1-x} \mathrm{Ga}_{x}$ As dots with high gallium content, which are larger, and show a stronger polarization anisotropy $\left(0.2\right.$ for $\operatorname{In}_{0.5} \mathrm{Ga}_{0.5}$ AsQD's, $<0.01$ for InAs QD's). On the other hand, also the shape of the dots might change with the gallium content. The only clear distinction can be made for the LCQD's, having a highly polarized emission along the coalescence direction [1-10] parallel to the sample surface, which is due to shape anisotropy. In the 10-layer sample, $P$ of the lower energy PL band is small compared to the main PL band [see Fig. 4(a)]. Since the lower energy band corresponds to optical transitions of larger QD's, this contradicts on influence of piezoelectric fields. We are thus led to assume that a shape change of the corresponding QD's plays an important role in our sample system.

The high values of in-plane polarization detected along the [110] direction (second row of Fig. 6) for both samples are thus tentatively assigned to shape elongation and lateral coupling along the [1-10] direction. Detecting in the [1-10] direction, the 10-layer sample shows a polarization mainly along the sample surface with a small out-of-plane component. This is reversed for the 20-layer sample. We attribute this to an increased vertical coupling due to an increasing size of the QD's at constant spacer thickness, a behavior that gives evidence that the VCQD's in the 20-layer sample are more "wirelike", instead of "dotlike."

\section{CONCLUSIONS}

In summary, we studied the polarization anisotropy of vertically coupled quantum dots. Highly polarized luminescence was observed, and ascribed mainly to shape anisotropy and lateral coupling. Moreover, a modification of the polarization from TE to TM with increasing number of layers was found, and attributed to increasing vertical coupling. However, only a detailed theoretical study of the transitions together with a direct correlation of the emission lines with measured dot shapes can give a definite answer about the microscopic origin of the observed behavior. In applications, the polarization control is essential to get either depolarized or TM-polarized emission via coupling of OD's from GaAsbased lasers in the important spectral range. Strongly surface-polarized PL can help to reach reliable polarization control in surface-emitting lasers.

\section{ACKNOWLEDGMENTS}

This work was supported by the Deutsche Forschungsgemeinschaft and by the Danish Ministries of Research and Education in the framework of CNAST. The work was also partly supported by INTAS, RFBR, and the Volkswagen Foundation. P. Y. is grateful for financial support from the Danish Research Academy.
*Present address: Ørsted Laboratory, Niels Bohr Institute, Copenhagen University, DK-2100 Copenhagen $\phi$, Denmark.

†Present address: Lehrstuhl für Experimentelle Physik EIlb, Universität Dortmund, Otto-Hahn Str. 4, D-44221 Dortmund, Germany.

${ }^{1}$ J.-Y. Marzin, J.-M. Gerard, A. Izrael, D. Barrier, and G. Bastard, Phys. Rev. Lett. 73, 716 (1994).
${ }^{2}$ D. Leonard, M. Krishnamurthy, C. M. Reaves, S. P. Denbaars, and P. M. Petroff, Appl. Phys. Lett. 63, 3203 (1993).

${ }^{3}$ M. Grundmann, O. Stier, and D. Bimberg, Phys. Rev. B 52, 11969 (1995).

${ }^{4}$ L. Goldstein, F. Glas, J. Y. Marzin, M. N. Charasse, and G. LeRoux, Appl. Phys. Lett. 47, 1099 (1985).

${ }^{5}$ Q. Xie, A. Madhukar, P. Chen, and N. P. Kobayashi, Phys. Rev. 
Lett. 75, 2542 (1995).

${ }^{6}$ G. S. Solomon, J. A. Trezza, A. F. Marshall, and J. S. Harris, Jr., Phys. Rev. Lett. 76, 952 (1996).

${ }^{7}$ A. A. Darhuber, V. Holy, J. Stangl, G. Bauer, A. Krost, F. Heinrichsdorff, M. Grundmann, D. Bimberg, V. M. Ustinov, P. S. Kopeiev, A. O. Kosogov, and P. Wenner, Appl. Phys. Lett. 70, 955 (1997).

${ }^{8}$ W. Wu, J. R. Tucker, G. S. Solomon, and J. S. Harris, Jr., Appl. Phys. Lett. 71, 1083 (1997).

${ }^{9}$ R. W. Winkler, J. P. Kootthaus, and K. Ploog, Phys. Rev. Lett. 62, 1177 (1989).

${ }^{10}$ R. Notzel, N. N. Ledentsov, L. Daweritz, M. Hohenstein, and K. Ploog, Phys. Rev. Lett. 67, 3812 (1991).

${ }^{11}$ S. S. Li and J. B. Xia, Phys. Rev. B 50, 8602 (1994).

${ }^{12}$ J. B. Xia and K. Huang, Chin. J. Semicond. 8, 563 (1987).

${ }^{13}$ J. A. Brum, G. Bastard, L. L. Chang, and L. Esaki, Superlattices Microstruct. 3, 47 (1987).

${ }^{14}$ M. Yang and J. C. Sturm, Phys. Rev. B 56, 1973 (1997).

${ }^{15}$ M. V. Maximov, Yu. M. Shernyakov, A. F. Tsatsul'nikov, A. V. Lunev, A. V. Sakharov, V. M. Ustinov, A. Yu. Egorov, A. E. Zhukov, A. R. Kovsh, P. S. Kop'ev, L. V. Asryan, Zh. I. Alferov, N. N. Ledentsov, D. Bimberg, A. O. Kosogov, and P. Werner, J. Appl. Phys. 83, 5561 (1998).

${ }^{16}$ A. F. Tsatsul'nikov, A. Yu. Egorov, A. E. Zhukov, A. R. Kovsh, V. M. Ustinov, N. N. Ledentsov, M. V. Maximov, B. V. Volo- vik, A. A. Suvorova, N. A. Bert, and P. S. Kop'ev, Fiz. Tekh. Poluprovodu. 36, 851 (1997) [Semiconductors 31, 722 (1997)].

${ }^{17}$ Y. Nabetani, T. Ishikawa, S. Noda, and A. Sasaki, J. Appl. Phys. 76, 347 (1994).

${ }^{18}$ H. Saito, K. Nishi, S. Sugou, and Y. Sugimoto, Appl. Phys. Lett. 71, 590 (1997), and references therein.

${ }^{19}$ S. Noda, T. Abe, and M. Tamura, Phys. Rev. B 58, 7181 (1998).

${ }^{20}$ M. Henini, S. Sanguinetti, S. C. Fortina, E. Grilli, M. Guzzi, G. Panzarini, L. C. Andreani, M. D. Upward, P. Moriarty, P. H. Beton, and L. Eaves, Phys. Rev. B 57, R6815 (1998).

${ }^{21}$ N. N. Ledentsov, V. A. Shchukin, M. Grundmann, N. Kirstaedter, J. Böhrer, O. Schmidt, D. Bimberg, V. M. Ustinov, A. Yu Egorov, A. E. Zhukov, P. S. Kop'ev, S. V. Zaitsev, N. Yu. Gordeev, Zh. I. Alferov, A. I. Borovkov, A. O. Kosogov, S. S. Ruvimov, P. Werner, U. Gösele, and J. Heydenreich, Phys. Rev. B 54, 8743 (1996).

${ }^{22}$ H. Qiang, F. H. Pollak, and G. Hickman, Solid State Commun. 76, 1087 (1990).

${ }^{23}$ H. Qiang, F. H. Pollak, C. Mailhiot, G. D. Pettit, and J. M. Woodall, Phys. Rev. B 44, 9126 (1991).

${ }^{24}$ H. Qiang, F. H. Pollak, S. Kai, Y. Takiguchi, R. R. Alfano, S. F. Fang, and H. Morkoc, Appl. Phys. Lett. 60, 2651 (1992).

${ }^{25}$ H. Qiang, F. H. Pollak, and R. N. Sacks, Solid State Commun. 84, 51 (1992). 